\title{
Digital sieving of pervious concrete air voids using X-ray computed tomography
}

\author{
Ajay Shankar Jagadeesh, Ghim Ping Ong, and Yu-Min Su
}

\begin{abstract}
The design and quality assessment of the pervious concrete mixtures based on its structural and functional performance are greatly influenced by the microstructural properties of the internal pore structure. The main objective of this study is to investigate the internal pore structure properties of two different pervious concrete mixtures (gap graded mixtures with nominal maximum aggregate sizes including $9.5 \mathrm{~mm}$ and $12.5 \mathrm{~mm}$ ) using $X$-Ray computed tomography and digital image processing. Image segmentation algorithms based on the histogram and laboratory volumetric characteristics of the pervious concrete mixtures have been utilised for the CT scan images. The key microstructural parameters of the air voids such as effective porosity, pore volume distribution, surface area distribution, elongation, flatness and shape factor distributions of the two different mixtures. It is expected that the developed procedure will serve as a valuable tool with potential applications in the current design methods of the pervious concrete pavements.
\end{abstract}

Index Terms - pervious concrete, x-ray computed tomography, volumetric segmentation, air voids, digital sieving.

\section{INTRODUCTION}

$\mathbf{T}$ he increased concern in the recent years toward reducing the pollutants and the environment resulted in the usage of pervious concrete as the pavement surface layers[1]. Pervious pavement is defined as the special class of pavement comprising materials with sufficient interconnected voids different mixtures were analysed and the results were compared for the ranging from 15 to $35 \%$ to allow water to pass through. The benefits of using the pervious pavement include the increase in the skid resistance and reduction in hydroplaning, storm water run-off and tire/road noise. The size and shape characteristics of the pervious concrete air voids have a significant influence on the water permeability and acoustic absorption characteristics [1].

In recent years, significant efforts have been made in analysing the infrastructure materials using CT scanning and digital image processing. Yun et al. [2] and Kim et al. [3] studied the equivalent pore diameters and paste void spacing of the hardened cement paste using CT scanning. Gruber et al. [4] examined the anisotropic permeability, pore size distribution, ice formation in the internal pore network of hot

A.S. Jagadeesh is with the Department of Civil and Environmental Engineering, National University of Singapore, 117576, Singapore (e-mail: ceeongr@nus.edu.sg)

G.P. Ong is with the Department of Civil and Environmental Engineering, National University of Singapore, 117576, Singapore

Y. Su is with the Department of Civil Engineering, National Kaohsiung University of Applied Sciences, Sanmin, Kaohsiung 80778, Taiwan. mix asphalt using CT scanning. Gao et al. [5] investigated the size, shape and distribution characteristics of the coarse aggregates in the cold recycling mixes using $\mathrm{CT}$ scanning. Kuang et al. [6] investigated the pore network properties of pervious concrete such as pore size distribution, porosity, tortuosity and specific surface area using X-Ray computed tomography. Shaheen et al. [7] developed a framework for evaluated the fatigue characteristics of hot mix asphalt using the pore volume distributions obtained from CT scanning. Abera et al., [8] investigated the effectiveness of global thresholding algorithms for different porous media including pervious concrete using void ratio. Zhang et al. [9] studied the pore size distributions of the various pervious concrete mixtures using watershed segmentation algorithm. Jagadeesh et al. [10] studied the effect of global thresholding algorithms on discharge and volumetric characteristics of the pervious concrete samples. The current paper analyses the effect of different segmentation algorithms on the digital sieving of pervious concrete air voids based on size and shape characteristics using CT scanning and digital image processing.

\section{MATERIALS}

To analyse the internal pore characteristics of the different mixtures, two pervious concrete mixtures $\mathrm{P} 1$ and $\mathrm{P} 2$ with the nominal maximum aggregate size of $9.5 \mathrm{~mm}$ and $12.5 \mathrm{~mm}$ respectively and the design porosity of $26.5 \%$ were produced. The siliceous aggregates of specific gravity 2.64 and percent absorption $1.35 \%$ and the binding agent ASTM Type I cement was used for the mixtures P1 and P2. The water-cement, cement-aggregate and superplasticizer-cement ratio used for the mixtures are $0.300,0.222$ and 0.006 respectively. Pervious concrete samples of $150 \mathrm{~mm}$ diameter and $250 \mathrm{~mm}$ height were casted in the laboratory and cured for 28 days in the limewater tank.

\section{X-RAY COMPUTED TOMOGRAPHY}

The medical X-Ray CT scanner of Somatom Emotion 16channel with an output voltage of $110 \mathrm{kV}$ energy was used to obtain the pore network structure of the pervious concrete samples P1 and P2. A total of 300 section images at the vertical interval of $0.7 \mathrm{~mm}$ has been obtained for the pixel size $1024 \times 1024$. The Simpleware ScanIP software was used in the conversion of X-Ray absorption coefficients for the voxels to the 16-bit greyscale intensities varying from 0 to 65535 , followed by image filtering using convolution kernel and segmentation into binary images using thresholding algorithms. Thresholding of air voids based on grey scale 
intensities was carried out using the following segmentation algorithms for pervious concrete mixtures $\mathrm{P} 1$ and P2. The watershed segmentation algorithm has been used to divide the interconnected pore structure into several pores based on the image background gradient. The cropped pervious concrete cubes of $100 \mathrm{~mm} \times 100 \mathrm{~mm} \times 100 \mathrm{~mm}$ were used for the further analysis.

\section{A. Otsu Trilevel Algorithm}

The Otsu trilevel algorithm [11] is used to segment the pervious concrete images into three phases such as aggregate, cement and air voids phase. In this algorithm, the two optimal thresholds for air void-cement and cement-aggregate are obtained by minimising the weighted within class variance or maximising the between class variance of the foreground and background classes in the image histogram. The objective function of the Otsu trilevel algorithm is given by

$$
\sigma_{b}^{2}=w_{0}\left(\mu_{0}-\mu_{T}\right)^{2}+w_{1}\left(\mu_{1}-\mu_{T}\right)^{2}+w_{2}\left(\mu_{2}-\mu_{T}\right)^{2}
$$

$\sigma_{b}^{2}$ is the between class variance, $w_{0}, w_{1}$ and $w_{2}$ are the total probabilities of intensities in each class, $\mu_{0}, \mu_{1}, \mu_{2}$ are the mean value of intensities in each class and $\mu_{T}$ is the mean value of intensities in the total histogram.

\section{B. Volumetric Based Global Minima Algorithm}

The volumetric algorithm [12] is used to segment the pervious concrete images into two phases such as solid and air voids phase. In this method, the threshold of air voids is obtained by minimising the absolute error of the experimental and image-based interconnected air void content. This method requires the laboratory determination of the percentage of interconnected air voids. The percentage of interconnected air voids or effective porosity in pervious concrete mixtures were assessed using Equation (2)

$$
n_{\text {eff }}=1-\frac{W_{2}-W_{1}}{\rho_{w} V}
$$

where $W_{1}$ and $W_{2}$ are the weight of the sample in water and in surface dry condition, $\rho_{w}$ is the density of the water and $V$ is the bulk volume of the sample, including the solid and void components.

\section{Otsu bilevel algorithm}

The Otsu bilevel algorithm [11] is used to segment the pervious concrete images into two phases such as solid and air voids phase. In this algorithm, the optimal threshold for air voids-solid phase is obtained by minimising the weighted within class variance or maximising the between class variance of the foreground and background classes in the image histogram. The objective function of the Otsu bilevel algorithm is given by

$$
\sigma_{b}^{2}=w_{0}\left(\mu_{0}-\mu_{T}\right)^{2}+w_{1}\left(\mu_{1}-\mu_{T}\right)^{2}
$$

where $\sigma_{b}^{2}$ is the between class variance, $w_{0}$ and $w_{1}$ are the total probabilities of intensities in each class, $\mu_{0}, \mu_{1}$ are the mean value of intensities in each class and $\mu_{T}$ is the mean value of intensities in the total histogram.

\section{RESUlTS AND DISCUSSION}

The three threshold segmentation techniques were applied to $\mathrm{X}$-Ray CT scan images of two different pervious concrete mixtures P1 and P2 and Table I shows the air void upper threshold values for the different algorithms. Fig. 1 and Fig. 2 shows the raw slice of the mixtures $\mathrm{P} 1$ and $\mathrm{P} 2$ along with the air voids segmented slices (white in colour) using different algorithms. Fig. 3 and Fig. 4 shows the three-dimensional pore network structure of the pervious concrete mixtures P1 and P2 using volumetric segmentation and volumetric-watershed segmentation algorithms.

TABLE I

PERVIOUS CONCRETE AIR VOID THRESHOLDS USING DIFFERENT SEGMENTATION ALGORITHMS

\begin{tabular}{ccc}
\multicolumn{2}{c}{ SEGMENTATION ALGORITHMS } \\
\hline Segmentation algorithms & $\begin{array}{c}\text { Air void upper } \\
\text { threshold values for } \\
\text { mixtures }\end{array}$ \\
\cline { 2 - 3 } Otsu trilevel & 1471 & 1168 \\
Volumetric & 1647 & 1694 \\
Otsu bilevel & 1678 & 1792 \\
\hline
\end{tabular}

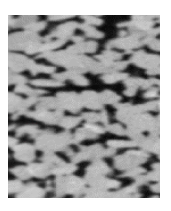
(a) Raw
image

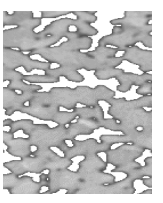

(b) Otsu trilevel

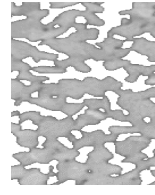

(c) Volumetri

c

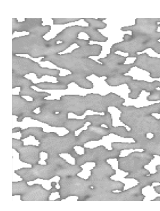

(d) Otsu

Fig. 1. Comparison of pervious concrete P1 raw image with different thresholding algorithms

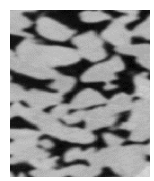

(a)

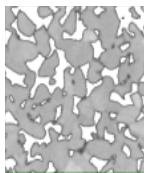

(b)

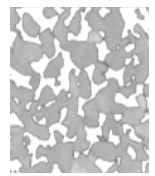

(c)

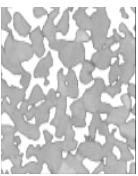

(d)

Raw image Otsutrilevel Volumetric Otsu bilevel

Fig. 2. Comparison of pervious concrete P2 raw image with different thresholding algorithms

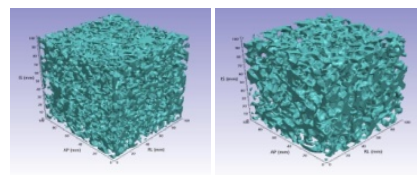

(a)

(b)

Fig. 3. Volumetric based pore network structure of pervious concrete mixtures P1 and P2 


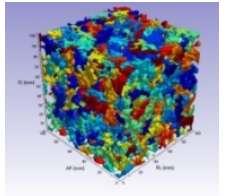

(a) P1

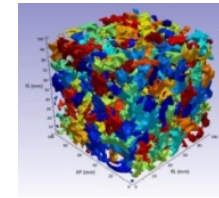

(b) P2
Fig. 4. Volumetric-Watershed based pore network structure of pervious concrete mixtures $\mathrm{P} 1$ and $\mathrm{P} 2$

\section{A. Effective Porosity}

Fig. 5 shows the effect of threshold on the effective porosity along with the laboratory effective porosities for the mixtures P1 and P2. It can be seen that, as the threshold value increases, the amount of air void voxels increases and hence the effective porosity value increases. The percentage error in the effective porosity using different segmentation algorithms are nearly $0 \%$ for volumetric algorithm due to calibration, less than $5 \%$ for Otsu bilevel and more than $20 \%$ for Otsu trilevel algorithm.

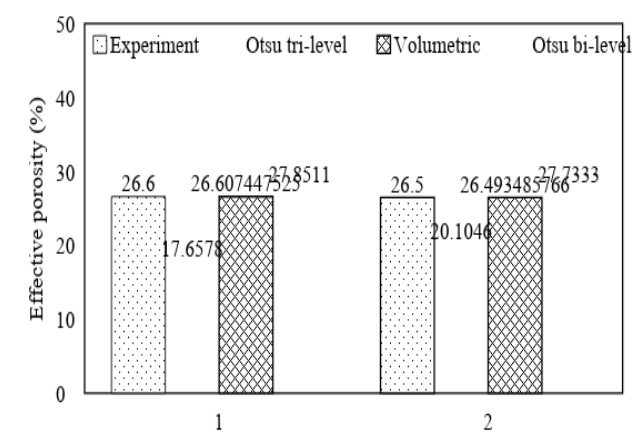

Fig. 5. Effect of threshold on effective porosity (\%)

\section{B. Pore Volume And Surface Area Distribution}

Fig. 6(a) shows the effect of threshold on the pore volume distribution for the pervious concrete mixtures P1 and P2. The variation in the distribution of the pore volume with respect to the different threshold values and different mixtures can be further understood by comparing its percentage in each volume range as shown in Fig. 6(b). It can be seen that as the threshold value increases, the percentage of smaller pores $\left(<100 \mathrm{~mm}^{3}\right)$ decreases and the percentage of larger pores increases due to the increase in the air void voxels and the increased connectivity. Comparing the volumetric segmentation algorithms of the pervious concrete mixtures $\mathrm{P} 1$ and $\mathrm{P} 2$, it can be observed that with an increase in the nominal maximum aggregate size, the amount of smaller pores reduces and larger pores increases as expected. The percentage error in the pore volume distribution with respect to the experimental based volumetric segmentation method is found to be significantly more for Otsu trilevel and less than $5.2 \%$ for Otsu bilevel segmentation method. Fig.7 shows the effect of threshold on the pore surface area and the cumulative distributions on the pervious concrete mixtures P1 and P2. A similar trend on the threshold effect on pore surface area distribution to the pore volume distribution was observed.

\section{Pore Flatness Distribution}

Fig. 8(a) shows the effect of threshold on the pore flatness distribution for the pervious concrete mixtures P1 and P2. The variation in the distribution of the pore flatness with respect to the different threshold values and different mixtures can be further understood by comparing its percentage in each volume range as shown in Fig. 8b. It can be seen that as the threshold value increases, the percentage of air voids in the flatness range 0.6 to 0.8 increases for both the mixtures P1 and P2. The pore flatness in the range less than 0.6 has slightly higher air voids percent for the mixture P2 compared to P1 for all threshold values.

\section{Pore Elongation Distribution}

Fig. 9(a) shows the effect of threshold on the pore elongation distribution for the pervious concrete mixtures P1 and $\mathrm{P} 2$. The variation in the distribution of the pore elongation with respect to the different threshold values and different mixtures can be further understood by comparing its percentage in each volume range as shown in Fig. 9(b). It can be seen that as the threshold value increases, the percentage of air voids in the elongation range 0.6 to 0.8 increases for both the mixtures $\mathrm{P} 1$ and $\mathrm{P} 2$. In the elongation range less than 0.6, increase in the threshold results in the increase of air void percent for P2 mixture and decrease for P1 mixture. In the elongation range greater than 0.8 , increase in the threshold results in the increase of air void percent for P1 mixture and decrease for $\mathrm{P} 2$ mixture. The pore elongation in the range less than 0.6 has slightly higher air voids percent for the mixture $\mathrm{P} 2$ compared to $\mathrm{P} 1$ for all threshold values.

\section{E. Pore Shape Factor Distribution}

Fig. 10 shows the effect of threshold on the pore shape factor distribution and its range for the pervious concrete mixtures P1 and P2. It can be seen that as the threshold value increases, the percentage of air voids in the elongated range decreases for mixture P1 and increases for mixture P2. In the flatted air voids range, the increase of threshold results in the increase of air voids for the mixture P1 and decrease of air voids for the mixture P2. It can also be seen that the mixture P1 with the smaller nominal maximum aggregate size has the higher percentage of air voids in the elongation zone compared to the mixture with the larger nominal maximum aggregate size. The pore shape factor in the range greater than 2 has slightly higher air voids percent for the mixture P1 compared to P2 for all threshold values. Further study is required to understand the effect of global segmentation algorithms on the pore shape characteristics of the pervious concrete mixtures. 


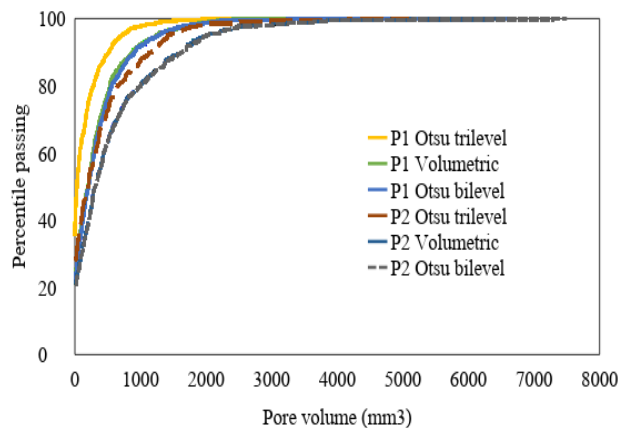

(a) Effect of threshold on the pore volume

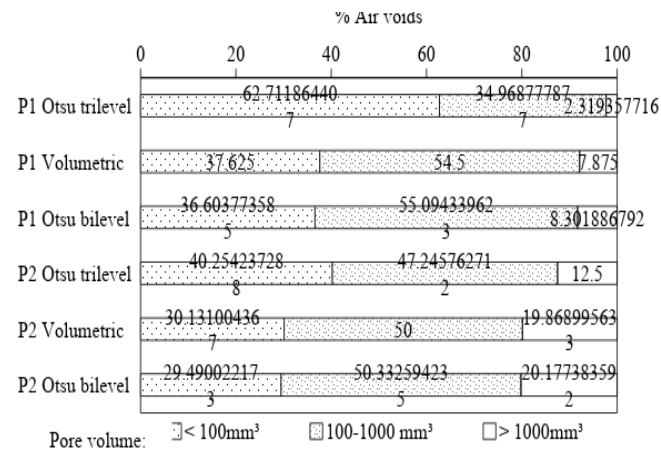

(b) Effect of threshold on the pore volume distribution Fig. 6. Effect of threshold on pore volume distribution and its range

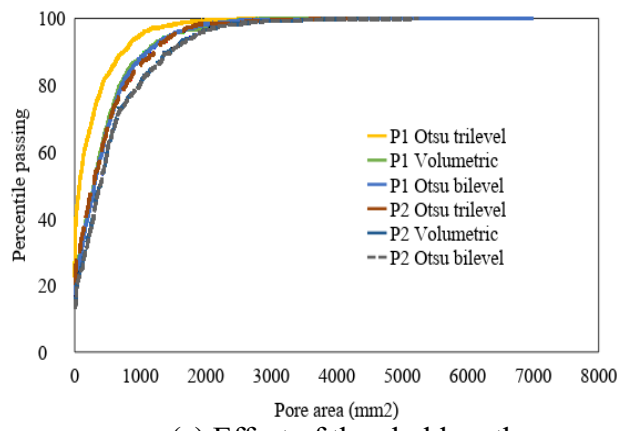

(a) Effect of threshold on the pore area

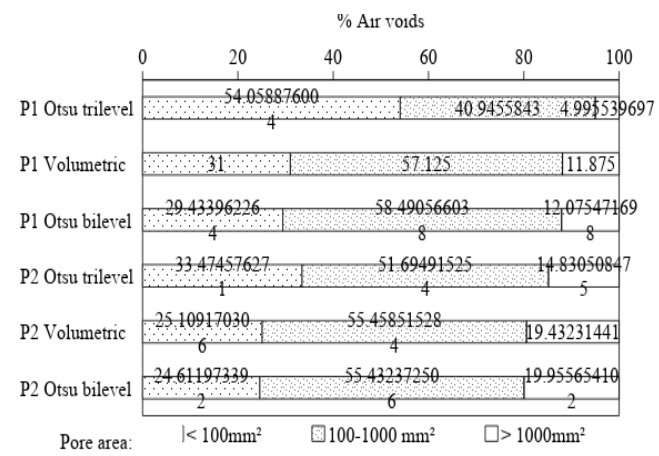

(b) Effect of threshold on the pore area distribution Fig. 7. Effect of threshold on pore surface area distribution and its range

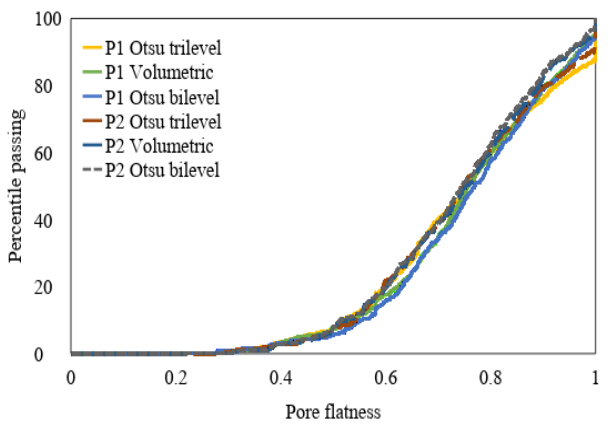

(a) Effect of threshold on the pore area

\begin{tabular}{|c|c|c|}
\hline 0 & 20 & 80 \\
\hline P1 Otsu trilevel & $\begin{array}{c}20.33898305 \\
1\end{array}$ & $\begin{array}{c}40.23193577 \\
2\end{array}$ \\
\hline P1 Volumetric & \begin{tabular}{l|l}
17.5 & $\because$
\end{tabular} & 40.875 \\
\hline P1 Otsu bilevel ${ }^{1}$ & $\begin{array}{c}5.84905660 \\
4\end{array}$ & $\begin{array}{c}42.38993710 \\
7\end{array}$ \\
\hline P2 Otsu trilevel & $\begin{array}{c}21.82203389 \\
8\end{array}$ & $\begin{array}{c}38.77118644 \\
1\end{array}$ \\
\hline P2 Volumetric & $\frac{19.65065502}{2}$ & $\begin{array}{c}39.30131004 \\
4\end{array}$ \\
\hline P2 Otsu bilevel & $\begin{array}{c}20.17738359 \\
2\end{array}$ & $\begin{array}{c}37.91574279 \\
4\end{array}$ \\
\hline $\begin{array}{r}\text { Pore flatness } \\
0.8\end{array}$ & $\square<$ & $>$ \\
\hline
\end{tabular}

(b) Effect of threshold on the pore flatness distribution

Fig. 8. Effect of threshold on pore flatness distribution and its range

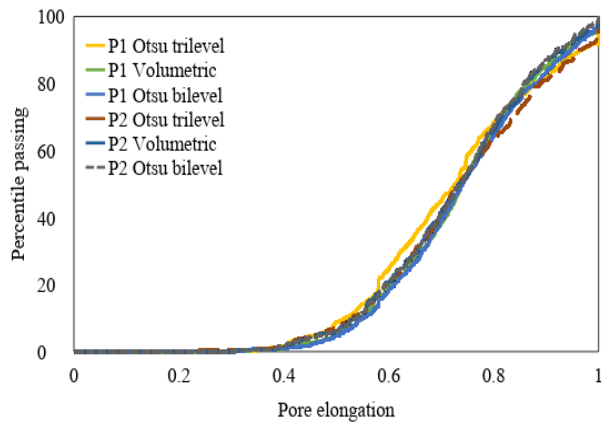

(a) Effect of threshold on the pore elongation

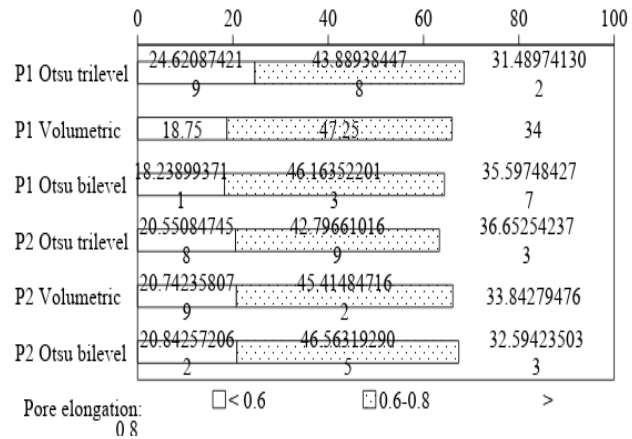

(b) Effect of threshold on the pore elongation distribution Fig. 9. Effect of threshold on pore elongation distribution and its range 


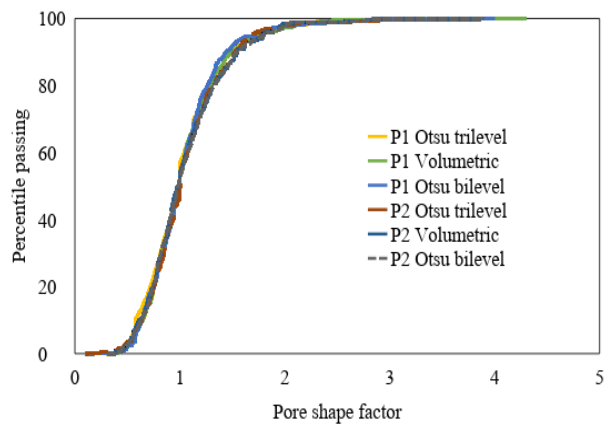

(a) Effect of threshold on the pore shape factor

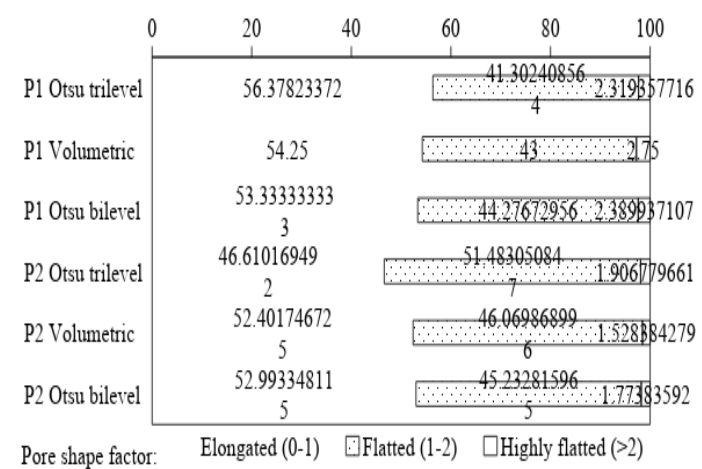

(b) Effect of threshold on the pore shape factor distribution

Fig. 10. Effect of threshold on pore shape factor distribution and its range.

\section{CONCLUSiOnS}

This study analyses the effect of global thresholding algorithms on the volumetric and pore network characteristics of the pervious concrete mixtures using X-Ray CT scanning. Two different mixtures and three different thresholding techniques were analysed numerically using the digital image processing algorithms. Based on the results of this study, the following conclusions may be drawn: (a) volumetric segmentation algorithm is considered to be predicting the effective porosity and pore characteristics more closely to the experimental results compared to the Otsu's bi-level and trilevel algorithms; (b) an increase in the nominal maximum aggregate size results in the reduction of percentage of smaller pores and increase of larger pores percent; (c) it can also be observed that the increase in the threshold results in the increase of larger pores percent and reduction in the smaller pores percent. Overall, it is expected that the present research will help in understanding the effect of global thresholding algorithms on the pore network characteristics of pervious concrete mixtures using digital image processing.

\section{REFERENCES}

[1] ACI 522R-2010. Report on Pervious Concrete. American Concrete Institute, 2010

[2] T.S. Yun, K.Y. Kim, J. Choo, and D.H. Kang. "Quantifying the distribution of paste-void spacing of hardened cement paste using X-ray computed tomography," Materials Characterization, 73, pp.137-143, 2012.
[3] K.Y. Kim, T.S Yun, J. Choo, D.H. Kang, and H.S. Shin. "Determination of air-void parameters of hardened cement-based materials using X-ray computed tomography,"cConstruction and Building Materials, 37, pp.93-101, 2012.

[4] I. Gruber, I. Zinovik, L. Holzer, A. Flisch, and L.D. Poulikakos. "A computational study of the effect of structural anisotropy of porous asphalt on hydraulic conductivity," Construction and Building Materials, 36, pp.66-77, 2012.

[5] L. Gao, F. Ni, H. Luo, H. Wang, and Y. Chen. "Evaluation of coarse aggregate in cold recycling mixes using X-Ray CT scanner and image analysis," Journal of Testing and Evaluation, 44(3), pp.1239-1249, 2014.

[6] X. Kuang, G. Ying, V. Ranieri, and J. Sansalone, "Examination of Pervious Pavement Pore Parameters with X-Ray Tomography," Journal of Environmental Engineering, 141(10), p.04015021, 2015.

[7] M. Shaheen, A. Al-Mayah, and S. Tighe, "A novel method for evaluating hot mix asphalt fatigue damage: X-ray computed tomography," Construction and Building Materials, 113, pp.121-133, 2016.

[8] K.A. Abera, K.N. Manahiloh, and M.M. Nejad, "The effectiveness of global thresholding techniques in segmenting two-phase porous media," Construction and Building Materials, 142, pp.256-267, 2017.

[9] J. Zhang, G. Ma, R. Ming, X. Cui, L. Li, and H. Xu, "Numerical study on seepage flow in pervious concrete based on 3D CT imaging," Construction and Building Materials, 161, pp.468-478, 2018.

[10] A. Jagadeesh, G.P. Ong, and Y.M. Su, "Porosity and permeability evaluation of pervious concrete using three dimensional X-ray computed tomography," In 4th International Conference on Transport Infrastructure, 2018.

[11] N. Otsu, "A threshold selection method from gray-level histograms," IEEE transactions on systems, man, and cybernetics, 9(1), pp.62-66, 1979.

[12] H.M. Zelelew, and A.T. Papagiannakis, "A volumetrics thresholding algorithm for processing asphalt concrete X-ray CT images," International journal of pavement engineering, 12(6), pp.543-551, 2011. 